\title{
Be wary of "prescribing" medical marijuana, CMA warns
}

$\mathrm{C}$ anadian physicians should be wary of "prescribing" medical marijuana under new regulations that come into effect on Apr. 1, 2014, says the president of the Canadian Medical Association.

"For the CMA, nothing has really changed," says Dr. Louis Hugo Francescutti. "Our stand has always been that there is insufficient scientific evidence to support the use of marijuana for clinical purposes."

In addition, he says, the regulatory colleges have indicated that they have concerns about patient safety and will be keeping a close eye on doctors who do "prescribe" the drug. "They are telling us to tread very gently. So if you are about to authorize access to marijuana you better really have the evidence that shows that it has some beneficial impact for your patient, because if something untoward happens you will be held to quite a high standard."

Under the previous program, the federal government provided a list of federally sanctioned medical conditions that would qualify a patient for medical marijuana. Doctors then completed a form confirming their patients' medical condition and Health Canada issued an authorization to possess marijuana. Francescutti says about 4000 to 5000 Canadian physicians have filed forms on behalf of patients.

With Health Canada's new Marihuana for Medical Purposes Regulations, the onus shifts from federal regulators to doctors and nurse practitioners who will decide which patients are eligible and what dose is appropriate. They will then write "medical documents" similar to prescriptions, authorizing patients to obtain marijuana from a federally licensed provider. Health Canada's role will be limited to licensing these marijuana providers.

The new regulations substantially change how marijuana is grown and distributed. Under the previous system, patients who received authorization from their doctor were permitted to

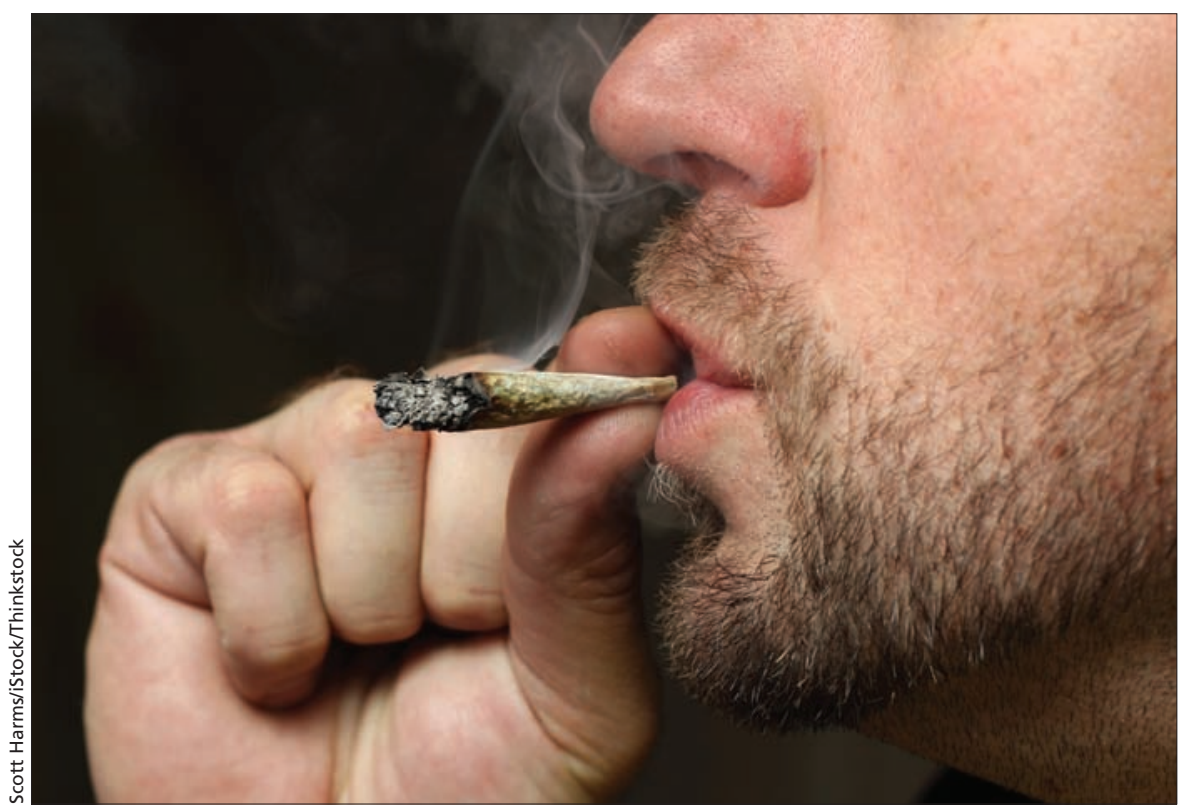

Under new federal regulations, marijuana producers will be required to list the level of active cannabinoids, so patients will know exactly what strength of weed they are getting.

possess and grow a small amount of marijuana for personal use, or designate someone to grow it for them. In a Mar. 14, 2014 media release, Health Canada states that this system was "open to serious abuse and had unintended consequences for public health, safety and security." As of Apr. 1, patients must obtain their marijuana by mail order from a licensed producer.

Marijuana is not an officially licensed or approved drug in Canada and Health Canada does not endorse its use. It became available for medicinal use following a 2000 Supreme Court of Canada ruling that Canadians should have reasonable access to a legal source of marijuana when authorized by a physician. It is most commonly prescribed for use during end-of-life care, and to treat severe pain or nausea associated with conditions such as multiple sclerosis, spinal injury, cancer and HIV/AIDS.

\section{Patient perspective}

The new system will have both advantages and disadvantages for patients, says Dr. Doug Smith, a specialist in physical medicine and rehabilitation in Fredericton, New Brunswick, who says he has signed authorizations for marijuana for about 500 patients over the past six or seven years.

On the upside, the time for the patient between gaining authorization from their physician and receiving the drug will be dramatically reduced, he says. In addition, the producers will be required to test their products for contaminants such as mould or pesticides, and to list the level of active cannabinoids, so patients will know exactly what strength of weed they are getting. The variability of home-grown plants is probably one reason doctors have been uncomfortable with authorizing marijuana use for their patients in the past, says Smith.

On the downside for the patient, the commercially grown marijuana will be more expensive than the homegrown variety - five to ten times more, or up to $\$ 7$ per gram, according to some estimates. And some patients may have to adjust their dosage as they get used to the strains offered by producers. 
Adam Greenblatt, executive director of the Medical Cannabis Access Society, a nonprofit dispensary in Montréal, Quebec, says he is pleased that Health Canada is expanding the number of strains available, but hopes that eventually other aspects, such as storefront dispensaries and cannabis extract products, will be legalized.

Greenblatt also insists that more research is vital to explore the many potential therapeutic properties of marijuana. "Cannabinoids could be their own domain of pharmacology."

Even now, doctors can be confident about the efficacy and safety of prescribing medical marijuana, says Greenblatt.
"Our understanding of its therapeutic effects is a lot more well-founded than the CMA will admit," he says. "Their major concern is about liability, but I think that has been largely exaggerated."

Smith says he was skeptical at first when patients started asking him for marijuana prescriptions, but was convinced when he witnessed the benefits in patients who had started using it on their own. "I was amazed at how effective and safe marijuana is for a lot of chronic conditions that have doctors wringing their hands in frustration, like fibromyalgia or spinal cord injury."

Authorizing medical marijuana is also a very low-risk proposition, says
Smith. "It's a very safe option for pain management; there has never been an attributable death." The same can't be said for even over-the-counter antiinflammatory medications like ibuprofen that can cause gastrointestinal bleeding.

Francescutti, though, still counsels caution until there is more evidence of the potential benefits and harms of the drug, and says doctors should not feel pressured by patients to prescribe it. "Physicians are under no obligation whatsoever to provide authorization for the use of medicinal marijuana." Brian Owens, St. Stephen, NB.

CMAJ 2014. DOI:10.1503/cmaj.109-4761 